\title{
Supporting Information \\ Regiospecific Structure, Degradation, and Functionalization of Polyperoxides Prepared from Sorbic Acid Derivatives with Oxygen.
}

\author{
Yuko Sugimoto, Shuji Taketani, Tomoaki Kitamura, Daisaku Uda, and Akikazu Matsumoto* \\ Department of Applied Chemistry, Graduate School of Engineering, Osaka City University, \\ Sugimoto, Sumiyoshi-ku, Osaka 558-8585, Japan \\ Corresponding author. FAX: +81-6-6605-2981. e mail: matsumoto@a-chem.eng.osaka-cu.ac.jp
}

\section{Monomer synthesis and spectral data}

Methyl sorbate (1a): liquid, ${ }^{1} \mathrm{H}$ NMR (400 MHz, $\left.\mathrm{CDCl}_{3}\right) \delta 7.26$ (dd, $J=15.2$ and $9.6 \mathrm{~Hz}, \mathrm{C} \underline{\mathrm{H}}=\mathrm{CHCO}$, 1H), 6.08-6.23 (m, $\left.\mathrm{CH}_{3} \underline{\mathrm{C}}=\mathrm{C} \underline{\mathrm{H}}, 2 \mathrm{H}\right), 5.77$ (d, $\left.J=15.2 \mathrm{~Hz}, \mathrm{CH}=\mathrm{C} \underline{\mathrm{HCO}}, 1 \mathrm{H}\right), 3.73\left(\mathrm{~s}, \mathrm{CO}_{2} \mathrm{CH}_{3}, 3 \mathrm{H}\right.$ ), 1.85 (d, $\left.J=5.9 \mathrm{~Hz}, \underline{\mathrm{CH}}_{3} \mathrm{CH}=\mathrm{CH}, 3 \mathrm{H}\right) ;{ }^{13} \mathrm{C} \mathrm{NMR}\left(100 \mathrm{MHz}, \mathrm{CDCl}_{3}\right) \delta 167.31(\mathrm{C}=\mathrm{O}), 144.84(\underline{\mathrm{CH}}=\mathrm{CHCO})$, $139.08\left(\mathrm{CH}_{3} \mathrm{CH}=\underline{\mathrm{CH}}\right), 129.52\left(\mathrm{CH}_{3} \underline{\mathrm{CH}}=\mathrm{CH}\right), 118.30(\mathrm{CH}=\underline{\mathrm{CHCO}}), 51.04\left(\mathrm{OCH}_{3}\right), 18.31\left(\underline{\mathrm{CH}}_{3} \mathrm{CH}=\mathrm{CH}\right)$.

$n$-Butyl sorbate (1b): liquid, ${ }^{1} \mathrm{H}$ NMR (400 MHz, $\left.\mathrm{CDCl}_{3}\right) \delta 7.24(\mathrm{dd}, J=9.6$ and $15.2 \mathrm{~Hz}$, $\mathrm{C} \underline{\mathrm{H}}=\mathrm{CHCO}, 1 \mathrm{H}), 6.13-6.18\left(\mathrm{~m}, \mathrm{CH}_{3} \mathrm{CH}=\mathrm{C} \underline{\mathrm{HCH}}=\mathrm{C} \underline{\mathrm{H}}, 2 \mathrm{H}\right), 5.77$ (d, $\left.J=15.6 \mathrm{~Hz}, \mathrm{CH}_{3} \mathrm{C} \underline{H}=\mathrm{CH}, 1 \mathrm{H}\right), 4.14$ (t, $J=6.6 \mathrm{~Hz}, \mathrm{CO}_{2} \mathrm{CH}_{2}, 2 \mathrm{H}$ ), 1.84 (d, J = 6.0 Hz, $\left.\underline{\mathrm{C}}_{3} \mathrm{CH}=\mathrm{CH}, 3 \mathrm{H}\right), 1.64$ (m, $\mathrm{CH}_{2} \underline{\mathrm{C}}_{2} \mathrm{CH}_{2} \mathrm{CH}_{3}, 2 \mathrm{H}$ ), 1.40 (m, $\mathrm{CH}_{2} \mathrm{CH}_{2} \underline{\mathrm{C}}_{2} \mathrm{CH}_{3}, 2 \mathrm{H}$ ), 0.94 (t, $J=7.4 \mathrm{~Hz}, \mathrm{CH}_{2} \underline{\mathrm{C}}_{3}, 3 \mathrm{H}$ ); ${ }^{13} \mathrm{C}$ NMR $\left(100 \mathrm{MHz}, \mathrm{CDCl}_{3}\right) \delta 166.28$ $(\mathrm{C}=\mathrm{O}), 144.13(\underline{\mathrm{CH}}=\mathrm{CHCO}), 138.26\left(\mathrm{CH}_{3} \mathrm{CH}=\underline{\mathrm{CH}}\right), 129.41\left(\mathrm{CH}_{3} \underline{\mathrm{CH}}=\mathrm{CH}\right), 118.66(\mathrm{CH}=\underline{\mathrm{CHCO}}), 63.28$ $\left(\mathrm{CO}_{2} \underline{\mathrm{CH}}_{2}\right), 30.32$ and $18.67\left(\mathrm{CH}_{2}\right), 17.94\left(\underline{\mathrm{CH}}_{3} \mathrm{CH}=\mathrm{CH}\right), 13.14\left(\mathrm{CH}_{2} \mathrm{CH}_{3}\right)$.

Decyl sorbate (1c): liquid, ${ }^{1} \mathrm{H}$ NMR (400 MHz, $\left.\mathrm{CDCl}_{3}\right) \delta 7.24$ (dd, $J=9.6$ and $15.2 \mathrm{~Hz}, \mathrm{C} \underline{\mathrm{H}}=\mathrm{CHCO}$, $1 \mathrm{H}), 6.08-6.17\left(\mathrm{~m}, \mathrm{CH}_{3} \mathrm{CH}=\mathrm{C} \underline{\mathrm{HCH}}=\mathrm{C} \underline{\mathrm{H}}, 2 \mathrm{H}\right), 5.77$ (d, $\left.J=15.6 \mathrm{~Hz}, \mathrm{CH}_{3} \underline{\mathrm{C}}=\mathrm{CH}, 1 \mathrm{H}\right), 4.12(\mathrm{t}, J=6.8 \mathrm{~Hz}$, $\mathrm{CO}_{2} \mathrm{CH}_{2}, 2 \mathrm{H}$ ), 1.84 (d, $\left.J=6.0 \mathrm{~Hz}, \mathrm{C}_{3} \mathrm{CH}=\mathrm{CH}, 3 \mathrm{H}\right), 1.65$ (m, $\left.\mathrm{CO}_{2} \mathrm{CH}_{2} \underline{\mathrm{C}}_{2}, 2 \mathrm{H}\right), 1.26\left(\mathrm{~m}, \mathrm{CH}_{2}, 14 \mathrm{H}\right)$, 0.88 (t, $\left.J=6.8 \mathrm{~Hz}, \mathrm{CH}_{2} \mathrm{CH}_{3}, 3 \mathrm{H}\right) ;{ }^{13} \mathrm{C}$ NMR $\left(100 \mathrm{MHz}, \mathrm{CDCl}_{3}\right) \delta 166.53(\mathrm{C}=\mathrm{O}), 144.25$ ( $\left.\mathrm{CH}=\mathrm{CHCO}\right)$, $\left.138.33\left(\mathrm{CH}_{3} \mathrm{CH}=\underline{\mathrm{CH}}\right), 129.54\left(\mathrm{CH}_{3} \underline{\mathrm{CH}}=\mathrm{CH}\right), 119.81(\mathrm{CH}=\underline{\mathrm{CHCO}}), 63.78\left(\mathrm{CO}_{2} \underline{\mathrm{CH}}\right)_{2}\right), 31.57,29.24,25.63$, and 22.32 $\left(\mathrm{CH}_{2}\right), 18.15\left(\mathrm{CH}_{3} \mathrm{CH}=\mathrm{CH}\right), 13.74\left(\mathrm{CH}_{2} \underline{\mathrm{CH}}_{3}\right)$.

Tetradecyl sorbate (1d): liquid, ${ }^{1} \mathrm{H}$ NMR $\left(400 \mathrm{MHz}, \mathrm{CDCl}_{3}\right) \delta 7.24(\mathrm{dd}, J=10.0$ and $15.6 \mathrm{~Hz}$, $\mathrm{C} \underline{\mathrm{H}}=\mathrm{CHCO}, 1 \mathrm{H}), 6.09-6.21\left(\mathrm{~m}, \mathrm{CH}_{3} \mathrm{CH}=\mathrm{C} \underline{\mathrm{HCH}}=\mathrm{C} \underline{\mathrm{H}}, 2 \mathrm{H}\right), 5.77$ (d, $\left.J=15.2 \mathrm{~Hz}, \mathrm{CH}_{3} \mathrm{C} \underline{H}=\mathrm{CH}, 1 \mathrm{H}\right), 4.12$ (t, $\left.J=6.6 \mathrm{~Hz}, \mathrm{CO}_{2} \mathrm{CH}_{2}, 2 \mathrm{H}\right), 1.84$ (d, $\left.J=5.6 \mathrm{~Hz}, \mathrm{C}_{3} \mathrm{CH}=\mathrm{CH}, 3 \mathrm{H}\right), 1.65\left(\mathrm{~m}, \mathrm{CO}_{2} \mathrm{CH}_{2} \mathrm{C}_{2}, 2 \mathrm{H}\right), 1.26$ (m, $\left.\mathrm{CH}_{2}, 22 \mathrm{H}\right), 0.88\left(\mathrm{t}, J=6.8 \mathrm{~Hz}, \mathrm{CH}_{2} \mathrm{CH}_{3}, 3 \mathrm{H}\right) ;{ }^{13} \mathrm{C} \mathrm{NMR}\left(100 \mathrm{MHz}, \mathrm{CDCl}_{3}\right) \delta 167.25(\mathrm{C}=\mathrm{O}), 144.68$, $(\underline{\mathrm{CH}}=\mathrm{CHCO}), 138.95\left(\mathrm{CH}_{3} \mathrm{CH}=\underline{\mathrm{CH}}\right), 129.70\left(\mathrm{CH}_{3} \underline{\mathrm{CH}}=\mathrm{CH}\right), 118.71(\mathrm{CH}=\underline{\mathrm{CHCO}}), 64.26\left(\mathrm{CO}_{2} \underline{\mathrm{CH}}_{2}\right), 31.83$, 29.60, 28.61, 25.86, and $22.60\left(\mathrm{CH}_{2}\right), 18.49\left(\mathrm{CH}_{3} \mathrm{CH}=\mathrm{CH}\right), 14.10\left(\mathrm{CH}_{2} \mathrm{CH}_{3}\right)$.

Octadecyl sorbate (1e): needles, mp $42-43^{\circ} \mathrm{C},{ }^{1} \mathrm{H}$ NMR (400 MHz, $\left.\mathrm{CDCl}_{3}\right) \delta 7.27$ (dd, $J=15.2$ and 
$9.6 \mathrm{~Hz}, \mathrm{C} \underline{H}=\mathrm{CHCO}, 1 \mathrm{H}), 6.09-6.23\left(\mathrm{~m}, \mathrm{CH}_{3} \mathrm{C} \underline{\mathrm{H}}=\mathrm{C} \underline{\mathrm{HCH}}=\mathrm{CH}, 2 \mathrm{H}\right), 5.78$ (d, $\left.J=15.2 \mathrm{~Hz}, \mathrm{CH}=\mathrm{C} \underline{H C O}, 1 \mathrm{H}\right)$, 4.13 (t, $\left.J=6.8 \mathrm{~Hz}, \mathrm{CO}_{2} \mathrm{CH}_{2}, 2 \mathrm{H}\right), 1.86$ (d, $\left.J=5.6 \mathrm{~Hz}, \underline{\mathrm{C}}_{3} \mathrm{CH}=\mathrm{CH}, 3 \mathrm{H}\right), 1.62\left(\mathrm{~m}, \mathrm{CH}_{2}, 2 \mathrm{H}\right), 1.2-1.4$ (m, $\left.\mathrm{CH}_{2}, 30 \mathrm{H}\right), 0.88\left(\mathrm{t}, J=6.8 \mathrm{~Hz}, \mathrm{CH}_{2} \mathrm{CH}_{3}, 3 \mathrm{H}\right) ;{ }^{13} \mathrm{C} \mathrm{NMR}\left(100 \mathrm{MHz}, \mathrm{CDCl}_{3}\right) \delta 167.40(\mathrm{C}=\mathrm{O}), 144.82$ $(\underline{\mathrm{CH}}=\mathrm{CHCO}), 139.15\left(\mathrm{CH}_{3} \mathrm{CH}=\underline{\mathrm{CHCH}}=\mathrm{CH}\right), 129.77\left(\mathrm{CH}_{3} \underline{\mathrm{CH}}=\mathrm{CH}\right), 119.02(\mathrm{CH}=\underline{\mathrm{CHCO}}), 64.40\left(\mathrm{OCH}_{2}\right)$, 31.90, 29.68, 29.56, 29.50, 29.35, 29.25, 28.67, 25.93, and $22.67\left(\mathrm{CH}_{2}\right), 18.62\left(\mathrm{CH}_{3} \mathrm{CH}=\mathrm{CH}\right), 14.10$ $\left(\mathrm{CH}_{2} \underline{\mathrm{CH}}\right)_{3}$.

$N$-n-Propylsorbamide (2a): powder, mp 86-88 ${ }^{\circ} \mathrm{C},{ }^{1} \mathrm{H}$ NMR (400 MHz, $\left.\mathrm{CDCl}_{3}\right) \delta 7.18$ (dd, $J=10.4$ and $15.2 \mathrm{~Hz}, \mathrm{C} \underline{\mathrm{H}}=\mathrm{CHCO}, 1 \mathrm{H}), 6.04-6.18\left(\mathrm{~m}, \mathrm{CH}_{3} \mathrm{C} \underline{\mathrm{H}}=\mathrm{C} \underline{\mathrm{H}}, 2 \mathrm{H}\right), 5.74(\mathrm{~d}, J=15.2 \mathrm{~Hz}, \mathrm{CH}=\mathrm{C} \underline{\mathrm{HCO}}, 1 \mathrm{H})$, 5.64 (broad, N태, 1H) 3.29 (q, $J=7.6 \mathrm{~Hz}, \underline{\mathrm{CH}}_{2} \mathrm{CH}_{2} \mathrm{CH}_{3}, 2 \mathrm{H}$ ), 1.83 (d, $J=6.4 \mathrm{~Hz}, \underline{\mathrm{C}}_{3} \mathrm{CH}=\mathrm{CH}, 3 \mathrm{H}$ ), 1.55 (m, $\mathrm{CH}_{2} \mathrm{C}_{2} \mathrm{CH}_{3}, 2 \mathrm{H}$ ), 0.93 (t, $J=7.2 \mathrm{~Hz}, \mathrm{CH}_{2} \mathrm{CH}_{2} \mathrm{C}_{3}, 3 \mathrm{H}$ ); ${ }^{13} \mathrm{C}$ NMR $\left(100 \mathrm{MHz}, \mathrm{CDCl}_{3}\right.$ ) $\delta 166.43$ $(\mathrm{C}=\mathrm{O}), 140.83$ ( $\underline{\mathrm{CH}}=\mathrm{CHCO}), 137.37\left(\mathrm{CH}_{3} \mathrm{CH}=\underline{\mathrm{CH}}\right), 129.65\left(\mathrm{CH}_{3} \underline{\mathrm{CH}}=\mathrm{CH}\right), 121.71(\mathrm{CH}=\underline{\mathrm{CHCO}}), 41.25$ $\left.\left(\mathrm{CH}_{2} \mathrm{CH}_{2} \mathrm{CH}_{3}\right), 22.83\left(\mathrm{CH}_{2} \mathrm{CH}_{2} \mathrm{CH}_{3}\right), 18.47\left(\mathrm{CH}_{3} \mathrm{CH}=\mathrm{CH}\right), 11.35\left(\mathrm{CH}_{2} \mathrm{CH}_{2} \underline{\mathrm{CH}}\right)_{3}\right)$.

$N$-tert-Butylsorbamide (2b): needles, mp $97-98^{\circ} \mathrm{C},{ }^{1} \mathrm{H}$ NMR (400 MHz, $\mathrm{CDCl}_{3}$ ) $\delta 7.13$ (dd, $J=10.4$ and $14.8 \mathrm{~Hz}, \mathrm{C} \underline{\mathrm{H}}=\mathrm{CHCO}, 1 \mathrm{H}), 6.18-6.25\left(\mathrm{~m}, \mathrm{CH}_{3} \underline{\mathrm{C}}=\mathrm{C} \underline{\mathrm{H}}, 2 \mathrm{H}\right), 5.67$ (d, $\left.J=15.2 \mathrm{~Hz}, \mathrm{CH}=\mathrm{C} \underline{\mathrm{HCO}}, 1 \mathrm{H}\right)$,

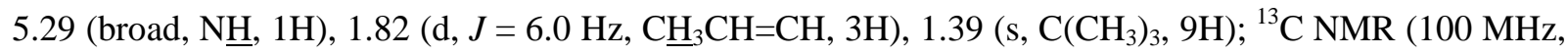
$\left.\mathrm{CDCl}_{3}\right) \delta 165.77(\mathrm{C}=\mathrm{O}), 139.61(\underline{\mathrm{CH}}=\mathrm{CHCO}), 136.20\left(\mathrm{CH}_{3} \mathrm{CH}=\underline{\mathrm{CH}}\right), 129.56\left(\mathrm{CH}_{3} \underline{\mathrm{CH}}=\mathrm{CH}\right), 123.15$ $(\mathrm{CH}=\underline{\mathrm{CHCO}}), 50.74\left(\underline{\mathrm{C}}\left(\mathrm{CH}_{3}\right)_{3}\right), 28.51\left(\mathrm{C}\left(\underline{\mathrm{CH}}_{3}\right)_{3}\right), 18.13\left(\underline{\left.\mathrm{CH}_{3} \mathrm{CH}=\mathrm{CH}\right)}\right.$.

$N$-Benzylsorbamide (2c): needles, mp $131^{\circ} \mathrm{C},{ }^{1} \mathrm{H}$ NMR (400 MHz, $\left.\mathrm{CDCl}_{3}\right) \delta 7.35-7.20$ (m, $\mathrm{Ar}$ and $\mathrm{C} \underline{\mathrm{H}}=\mathrm{CHCO}, 6 \mathrm{H}$ ), 6.12 (m, $\left.\mathrm{CH}_{3} \mathrm{C} \underline{\mathrm{H}}=\mathrm{C} \underline{\mathrm{H}}, 2 \mathrm{H}\right), 5.77$ (broad, NH, 1H), 5.75 (d, $J=15.2 \mathrm{~Hz}, \mathrm{CH}=\mathrm{C} \underline{\mathrm{HCO}}$, $1 \mathrm{H}), 4.52\left(\mathrm{~d}, J=6.0 \mathrm{~Hz}, \mathrm{CH}_{2}, 2 \mathrm{H}\right), 1.83\left(\mathrm{~d}, J=6.0 \mathrm{~Hz}, \mathrm{CH}_{3} \mathrm{CH}=\mathrm{CH}, 3 \mathrm{H}\right) ;{ }^{13} \mathrm{C}$ NMR $\left(100 \mathrm{MHz}, \mathrm{CDCl}_{3}\right)$ $\delta 166.18(\mathrm{C}=\mathrm{O}), 141.40$ ( $\underline{\mathrm{CH}}=\mathrm{CHCO}), 137.78\left(\mathrm{CH}_{3} \mathrm{CH}=\underline{\mathrm{CH}}\right), 129.62\left(\mathrm{CH}_{3} \underline{\mathrm{CH}}=\mathrm{CH}\right), 121.31(\mathrm{CH}=\underline{\mathrm{CHCO}})$, 137.07, 135.28, 129.28, and $127.83(\mathrm{Ar}), 43.35\left(\mathrm{CH}_{2}\right), 18.54\left(\mathrm{CH}_{3} \mathrm{CH}=\mathrm{CH}\right)$.

$\mathrm{N}$-(1-phenylethyl)sorbamide (2d): powder, mp 82-83 ${ }^{\circ} \mathrm{C},{ }^{1} \mathrm{H}$ NMR $\left(400 \mathrm{MHz}, \mathrm{CDCl}_{3}\right) \delta 7.35-7.17$ (m, Ar and $\underline{\mathrm{CH}}=\mathrm{CHCO}), 6,12\left(\mathrm{~m}, \mathrm{CH}_{3} \mathrm{C} \underline{\mathrm{H}}=\mathrm{C} \underline{\mathrm{H}}, 2 \mathrm{H}\right), 5.72(\mathrm{~d}, J=15.2 \mathrm{~Hz}, \mathrm{CH}=\mathrm{C} \underline{\mathrm{HCO}}, 1 \mathrm{H}), 5.63$ (broad, NH, 1H), 5.24 (m, $\left.\mathrm{CH}(\mathrm{Ar}) \mathrm{CH}_{3}, 1 \mathrm{H}\right), 1.83$ (d, $\left.J=5.6 \mathrm{~Hz}, \mathrm{CH}_{3} \mathrm{CH}=\mathrm{CH}, 3 \mathrm{H}\right), 1.53$ (d, $J=6.8 \mathrm{~Hz}, \mathrm{CH}(\mathrm{Ar}) \underline{\mathrm{H}}_{3}$, $3 \mathrm{H}) ;{ }^{13} \mathrm{C}$ NMR (100 MHz, $\left.\mathrm{CDCl}_{3}\right) \delta 165.46$ (C=O), 141.32 ( $\left.\underline{\mathrm{CH}}=\mathrm{CHCO}\right), 137.68\left(\mathrm{CH}_{3} \mathrm{CH}=\underline{\mathrm{CH}}\right), 129.62$ $\left(\mathrm{CH}_{3} \underline{\mathrm{CH}}=\mathrm{CH}\right), 121.53(\mathrm{CH}=\underline{\mathrm{CHCO}}), 143.26,128.55,127.20$, and $126.18(\mathrm{Ar}), 48.65\left(\underline{\mathrm{CH}}(\mathrm{Ar}) \mathrm{CH}_{3}\right) 21.68$ (CH(Ar) $\left.\underline{\mathrm{CH}}_{3}\right), 18.51\left(\mathrm{CH}_{3} \mathrm{CH}=\mathrm{CH}\right)$.

$N$-Phenylsorbamide (2e): needles, mp $120-121^{\circ} \mathrm{C},{ }^{1} \mathrm{H}$ NMR $\left(400 \mathrm{MHz}, \mathrm{CDCl}_{3}\right) \delta 7.57$ (broad, NH, 1H), 7.36-7.09 (m, Ar and $\underline{\mathrm{CH}}=\mathrm{CHCO}, 6 \mathrm{H}), 6.18$ (m, $\left.\mathrm{CH}_{3} \mathrm{C} \underline{\mathrm{H}}=\mathrm{C} \underline{\mathrm{H}}, 2 \mathrm{H}\right), 5.89$ (d, $J=15.2 \mathrm{~Hz}, \mathrm{CH}=\mathrm{C} \underline{H C O}$, $1 \mathrm{H}), 1.87$ (d, $\left.J=5.6 \mathrm{~Hz}, \mathrm{CH}_{3} \mathrm{CH}=\mathrm{CH}, 3 \mathrm{H}\right) ;{ }^{13} \mathrm{C} \mathrm{NMR}\left(100 \mathrm{MHz}, \mathrm{CDCl}_{3}\right) \delta 164.65(\mathrm{C}=\mathrm{O}), 142.57$ ( $\underline{\mathrm{C}}=\mathrm{CHCO}), 138.14\left(\mathrm{CH}_{3} \mathrm{CH}=\underline{\mathrm{CH}}\right), 129.59\left(\mathrm{CH}_{3} \underline{\mathrm{CH}}=\mathrm{CH}\right), 121.69$ (CH=ㅡㅐCO), 138.14, 128.93, 124.14, 
and $119.90(\mathrm{Ar}), 18.62\left(\mathrm{CH}_{3} \mathrm{CH}=\mathrm{CH}\right)$.

$N, N$-Dimethylsorbamide (3a): needles, ${ }^{1} \mathrm{H}$ NMR (400 MHz, CDCl $) \delta 7.25$ (dd, $J=10.8$ and $15.2 \mathrm{~Hz}$, $\mathrm{C} \underline{\mathrm{H}}=\mathrm{CHCO}, 1 \mathrm{H}$ ), 6.23 (d, $J=15.2 \mathrm{~Hz}, \mathrm{CH}=\mathrm{C} \underline{\mathrm{HCO}}, 1 \mathrm{H}$ ), 6.04-6.25 (m, $\mathrm{CH}_{3} \underline{\mathrm{CH}}=\mathrm{C} \underline{\mathrm{H}}, 2 \mathrm{H}$ ), 3.01 and 3.08 (s, $\left.\mathrm{N}\left(\mathrm{CH}_{3}\right)_{2}, 6 \mathrm{H}\right), 1.84\left(\mathrm{~d}, \mathrm{~J}=6.4 \mathrm{~Hz}, \mathrm{CH}_{3} \mathrm{CH}=\mathrm{CH}, 3 \mathrm{H}\right), 1.39\left(\mathrm{~s}, \mathrm{C}\left(\mathrm{CH}_{3}\right)_{3}, 9 \mathrm{H}\right) ;{ }^{13} \mathrm{C} \mathrm{NMR}\left(100 \mathrm{MHz}, \mathrm{CDCl}_{3}\right)$ $\delta 166.91(\mathrm{C}=\mathrm{O}), 142.54$ ( $\underline{\mathrm{CH}}=\mathrm{CHCO}), 137.29\left(\mathrm{CH}_{3} \mathrm{CH}=\underline{\mathrm{CH}}\right), 130.08\left(\mathrm{CH}_{3} \underline{\mathrm{CH}}=\mathrm{CH}\right), 118.04$ (CH=ㅡHCO), 37.13 and $35.60\left(\mathrm{~N}\left(\mathrm{CH}_{3}\right)_{2}\right), 18.44\left(\underline{\mathrm{CH}}_{3} \mathrm{CH}=\mathrm{CH}\right)$.

$N, N$-Diphenylsorbamide (3b): liquid, ${ }^{1} \mathrm{H}$ NMR $\left(400 \mathrm{MHz}, \mathrm{CDCl}_{3}\right) \delta 7.88$ (d, $J=15.2 \mathrm{~Hz}, \mathrm{C} \underline{H}=\mathrm{CHCO}$, $1 \mathrm{H}$ ), 5.79 (d, $J=15.2 \mathrm{~Hz}, \mathrm{CH}=\mathrm{C} \underline{\mathrm{HCO}}, 1 \mathrm{H}$ ), 4.22 (q, $\left.J=7.2 \mathrm{~Hz}, \mathrm{OC}_{2} \mathrm{CH}_{3}, 2 \mathrm{H}\right), 1.79\left(\mathrm{~s}, \mathrm{CH}_{3}, 3 \mathrm{H}\right.$ ), 1.88 (s, $\left.\mathrm{C}_{3}, 3 \mathrm{H}\right), 1.97$ (s, $\left.\mathrm{CH}_{3}, 3 \mathrm{H}\right), 1.31$ (t, $\left.J=7.2 \mathrm{~Hz}, \mathrm{OCH}_{2} \underline{\mathrm{CH}}_{3}, 3 \mathrm{H}\right) ;{ }^{13} \mathrm{C} \mathrm{NMR}\left(100 \mathrm{MHz}, \mathrm{CDCl}_{3}\right) \delta 168.14$ $(\mathrm{C}=\mathrm{O}), 143.92(\underline{\mathrm{CH}}=\mathrm{CHCO}), 141.63\left(\left(\mathrm{CH}_{3}\right)_{2} \mathrm{C}=\underline{\mathrm{CCH}_{3}}\right), 125.87\left(\left(\mathrm{CH}_{3}\right)_{2} \underline{\mathrm{C}}=\mathrm{CCH}_{3}\right), 115.42(\mathrm{CH}=\underline{\mathrm{CHCO}})$, $60.07\left(\mathrm{OCH}_{2} \mathrm{CH}_{3}\right)$ 22.52, 20.92, and $\left.14.35\left(\left(\underline{\mathrm{CH}}_{3}\right)_{2} \mathrm{C}=\mathrm{C}_{\underline{C}}\right)_{3}\right), 14.05\left(\mathrm{OCH}_{2} \underline{\mathrm{CH}_{3}}\right)$.

(6-Hexa-2,4-dienoyloxy)-1,2,3,4-tetraacetylglucose (4a):

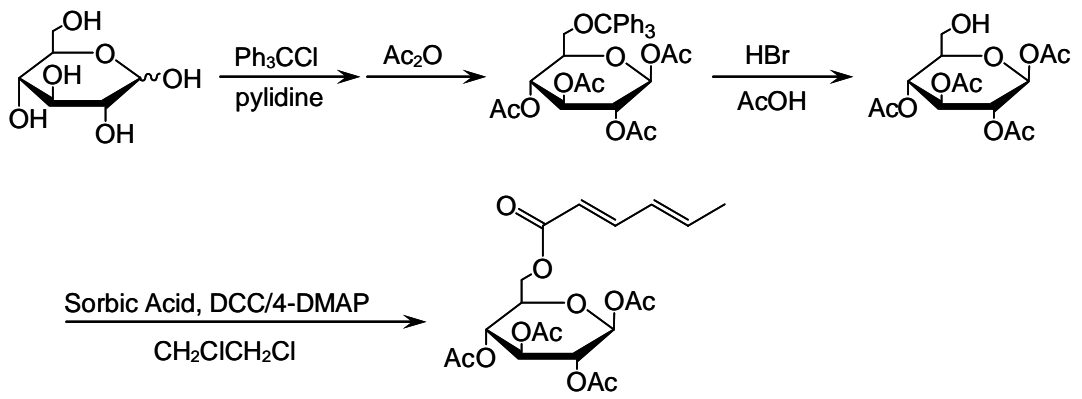

1,2,3,4-Tetraacetyl-6-triphenylmethylglucose. Triphenylmethyl chloride (6.8 g, $24 \mathrm{mmol}$ ) was added to $D$-(+)-glucose (5.4 g, $30 \mathrm{mmol}$ ) in pyridine, and stirred at room temperature for $24 \mathrm{~h}$ under a nitrogen atmosphere. Acetic anhydride (22 g, $0.21 \mathrm{~mol}$ ) was dropwose added over $30 \mathrm{~min}$, and then stirred for $6 \mathrm{~h}$. The solvent and an excess of acetic anhydride were removed under reduced pressure. The residue was dissolved in chloroform, and washed with $\mathrm{NaHCO}_{3}$, water, and brine. After drying over $\mathrm{Na}_{2} \mathrm{SO}_{4}$, the solvent was evaporated, followed by recrystallization from $n$-hexane. The pure 1,2,3,4-tetraacetyl-6-triphenylmethylglucose was isolated by recrystallization from a mixture of diethyl ether and $n$-hexane (1:1). Yield 40.4\%.

1,2,3,4-Tetraacetyl-6-hydroxyglucose. To 1,2,3,4-tetraacetyl-6-triphenylmethylglucose (3.4 g, 5.8 $\mathrm{mmol}$ ) in $30 \mathrm{~mL}$ of acetic acid, $2 \mathrm{~mL}$ of hydrobromic acid (48\%) was dropwise added at $10-15^{\circ} \mathrm{C}$, and stirred for $1 \mathrm{~min}$. After the precipitated triphenylmethyl bromide was filtered out, the filtrate was poured into $200 \mathrm{~mL}$ of water. After extraction with chloroform (50 mL x 4), the chloroform solution was washed 
several times with water, and then dried over $\mathrm{Na}_{2} \mathrm{SO}_{4}$. The solvent was evaporated and the residue was recrystallized from a mixture of diethyl ether and $n$-hexane (1:1). Yield 73.8\%.

(6-Hexa-2,4-dienoyloxy)-1,2,3,4-tetraacetylglucose. To the mixture of 1,2,3,4-tetraacetyl-6-hydroxyglucose (2.0 g, $5.7 \mathrm{mmol})$, sorbic acid ( $0.64 \mathrm{~g}, 5.7 \mathrm{mmol}$ ), and 4-dimethylaminopiridine (4-DMAP, $0.35 \mathrm{~g}$ ) in 1,2-dichloroethane, $N, N$-dicyclohexylcarbodimide (DCC, 1,2 g, $5.8 \mathrm{mmol}$ ) in 1,2-dichloroethane was dropwise added at $0^{\circ} \mathrm{C}$, and stirred at room temperature overnight. After removing the precipitated di(cyclohexyl)urea, the filtrate was concentrated. Silica gel column chromatography (chloroform/ethyl acetate $=10 / 1$ ) provided (6-hexa-2,4-dienoyloxy)-1,2,3,4-tetraacetylglucose (4a), followed by recrystallization from diethyl ether. Yield 61.5\%.

4a: powder; mp $120-121^{\circ} \mathrm{C} ;{ }^{1} \mathrm{H}$ NMR $\left(400 \mathrm{MHz}, \mathrm{CDCl}_{3}\right) \delta 7.31$ (m, $\left.\mathrm{C} \underline{H}=\mathrm{CHC}=\mathrm{O}, 1 \mathrm{H}\right), 6.20$ (m, $\left.\mathrm{CH}_{3} \mathrm{C} \underline{\mathrm{H}}=\mathrm{C} \underline{\mathrm{H}}, 2 \mathrm{H}\right), 5.81(\mathrm{~d}, J=14.8 \mathrm{~Hz}, \mathrm{CH}=\mathrm{C} \underline{\mathrm{HC}}=\mathrm{O}, 1 \mathrm{H}), 5.74(\mathrm{~d}, J=8.4 \mathrm{~Hz}, \mathrm{C} 1(\mathrm{H}), 1 \mathrm{H}), 5.28$ (t, $J=$ $9.6 \mathrm{~Hz}, \mathrm{C} 3(\mathrm{H}), 1 \mathrm{H}), 5.17(\mathrm{~m}, \mathrm{C} 2(\mathrm{H})$ and $\mathrm{C} 4(\mathrm{H}), 2 \mathrm{H}), 4.31\left(\mathrm{~m}, \mathrm{C} 6\left(\mathrm{H}_{2}\right), 2 \mathrm{H}\right), 3.91(\mathrm{~m}, \mathrm{C} 5(\mathrm{H}), 1 \mathrm{H})$, 2.12-2.02 (s, $\left.\mathrm{CH}_{3} \mathrm{CO}, 12 \mathrm{H}\right), 1.87$ (d, $J=5.2 \mathrm{~Hz}, \mathrm{CH}_{3} \mathrm{CH}=\mathrm{CH}, 3 \mathrm{H}$ ); ${ }^{13} \mathrm{C} \mathrm{NMR}\left(100 \mathrm{MHz}, \mathrm{CDCl}_{3}\right) \delta 170.1$, 169.3, 169.2, $168.9\left(\mathrm{CH}_{3} \underline{\mathrm{C}}=\mathrm{O}\right), 166.6(\mathrm{CH}=\mathrm{CH} \underline{\mathrm{C}}=\mathrm{O}), 146.1(\underline{\mathrm{CH}}=\mathrm{CHCO}), 140.2\left(\mathrm{CH}_{3} \mathrm{CH}=\underline{\mathrm{CH}}\right), 129.7$ $\left(\mathrm{CH}_{3} \underline{\mathrm{CH}}=\mathrm{CH}\right.$ ), 117.8 (CH=ㅡㅐCO), 91.6 (C1), 72.8 (C3), 72.7 (C5), 70.1 (C2), 67.9 (C4), 61.4 (C6), 20.8-20.4 ( $\left.\underline{\mathrm{CH}}_{3} \mathrm{CO}\right), 18.7\left(\mathrm{CH}_{3} \mathrm{CH}=\mathrm{CH}\right)$.

(1-Pyrenyl)methyl sorbate (4b): $\mathbf{4 b}$ was synthesized by a procedure similar to that for $\mathbf{4 a}$. To sorbic acid (0.5 g, $4.4 \mathrm{mmol})$, (1-pyrenyl)methanol (4.4 mmol), and 4-DMAP (4.1mmol) in 1,2-dichloroethane, DCC (1.0 g, $4.8 \mathrm{mmol})$ in 1,2-dichloroethane was dropwise added, and stirred at room temperature overnight. After work-up, the crude product was recrystallized from a mixture of 1,2-dichloroethane and $n$-hexane (1:1). Yield 84\%.

4b: powder; mp $113-114^{\circ} \mathrm{C}$; ${ }^{1} \mathrm{H}$ NMR (400 MHz, $\mathrm{CDCl}_{3}$ ) $\delta$ 8.39-8.01 (m, Ar, 9H), 7.31 (dd, $J=10.0$ $\mathrm{Hz}$ and $15.6 \mathrm{~Hz}, \mathrm{C} \underline{\mathrm{H}}=\mathrm{CHCO}, 1 \mathrm{H}), 6.13\left(\mathrm{~m}, \mathrm{CH}_{3} \mathrm{C} \underline{\mathrm{H}}=\mathrm{C} \underline{\mathrm{H}}, 2 \mathrm{H}\right), 5.91\left(\mathrm{~s}, \mathrm{CH}_{2}, 2 \mathrm{H}\right), 5.84(\mathrm{~d}, J=15.6 \mathrm{~Hz}$, $\mathrm{CH}=\mathrm{C} \underline{\mathrm{HCO}}, 1 \mathrm{H}), 1.82\left(\mathrm{~d}, J=6.0 \mathrm{~Hz}, \mathrm{CH}_{3} \mathrm{CH}=\mathrm{CH}, 3 \mathrm{H}\right) ;{ }^{13} \mathrm{C}$ NMR $\left(100 \mathrm{MHz}, \mathrm{CDCl}_{3}\right) \delta 167.5(\mathrm{C}=\mathrm{O})$, 145.9 ( $\underline{\mathrm{CH}}=\mathrm{CHCO}), 140.1\left(\mathrm{CH}_{3} \mathrm{CH}=\underline{\mathrm{CH}}\right), 132.0,131.5,131.0,130.0,129.8,129.4,128.4,128.0,127.9$, 127.6, 126.2. 125.7, 125.6, 125.1, 124.9, and 123.3 (Ar), $130.1\left(\mathrm{CH}_{3} \underline{\mathrm{CH}}=\mathrm{CH}\right), 118.9$ (CH=ㅡCO), 64.8 $\left(\mathrm{CH}_{2}\right), 19.0\left(\mathrm{CH}_{3} \mathrm{CH}=\mathrm{CH}\right)$.

4-(Phenylazophenyl) sorbate (4c): 4c was prepared similarly. Yield 64\%. powder; mp; $119-120^{\circ} \mathrm{C} ;{ }^{1} \mathrm{H}$ NMR (400 MHz, $\left.\mathrm{CDCl}_{3}\right) \delta$ 7.98-7.92, 7.54-7.44, and 7.31-7.26 (m, Ar and $\left.\underline{\mathrm{CH}}=\mathrm{CHCO}, 10 \mathrm{H}\right), 6.28$ (m, $\left.\mathrm{CH}_{3} \mathrm{C} \underline{\mathrm{H}}=\mathrm{C} \underline{\mathrm{H}}, 2 \mathrm{H}\right), 5.99$ (d, $\left.J=16.0 \mathrm{~Hz}, \mathrm{CH}=\mathrm{C} \underline{H C O}, 1 \mathrm{H}\right), 1.91$ (d, $\left.J=5.2 \mathrm{~Hz}, \mathrm{C}_{3} \mathrm{CH}=\mathrm{CH}, 3 \mathrm{H}\right) ;{ }^{13} \mathrm{C}$ NMR (100 MHz, $\left.\mathrm{CDCl}_{3}\right) \delta 165.3$ (C=O), 147.4 ( $\left.\underline{\mathrm{CH}}=\mathrm{CHCO}\right), 141.2\left(\mathrm{CH}_{3} \mathrm{CH}=\underline{\mathrm{CH}}\right), 152.9,152.5,150.1$, 131.0, 129.1, 124.0, 122.8, and 122.3 (Ar), $129.7\left(\mathrm{CH}_{3} \underline{\mathrm{CH}}=\mathrm{CH}\right), 117.6(\mathrm{CH}=\underline{\mathrm{CHCO}}), 18.8\left(\underline{\mathrm{CH}}_{3} \mathrm{CH}=\mathrm{CH}\right)$. 2-(Methacryloyloxy)ethyl sorbate (4d): Sorbic acid (5.6 g, $50 \mathrm{mmol})$, thionyl chloride (4 mL, 55 
mmol), and a catalytic amount of dimethylformamide in 1,2-dichloroethane were refluxed for $30 \mathrm{~min}$. The obtained sorbic acid chloride was allowed to react with 2-hydroxyethyl methacrylate (6.5 g, $50 \mathrm{mmol}$ ) without isolation and further purification. Silica gel column chromatography (ethyl acetate/n-hexane = 2/1) provided $\mathbf{4 d}$ in the yield of $56.8 \%$.

4d: liquid; ${ }^{1} \mathrm{H}$ NMR (400 MHz, $\mathrm{CDCl}_{3}$ ) $\delta 7.28$ (dd, $J=10.8$ and $15.2 \mathrm{~Hz}, \mathrm{C} \underline{H}=\mathrm{CHCO}, 1 \mathrm{H}$ ), 6.21-6.14 (m, $\mathrm{CH}_{3} \underline{\mathrm{CH}}=\mathrm{C} \underline{\mathrm{H}}$ and $\left.\mathrm{CCO}\left(\mathrm{CH}_{3}\right)=\underline{\mathrm{C}}_{1} \mathrm{H}_{2}, 3 \mathrm{H}\right), 5.79(\mathrm{~d}, J=15.6 \mathrm{~Hz}, \mathrm{CH}=\mathrm{C} \underline{\mathrm{HCO}}, 1 \mathrm{H}), 5.59$ (s, $\left.\mathrm{CCO}\left(\mathrm{CH}_{3}\right)=\mathrm{CH}_{1} \underline{\mathrm{H}}_{2}, 1 \mathrm{H}\right), 4.41-4.38\left(\mathrm{~m}, \underline{\mathrm{C}}_{2} \underline{\mathrm{C}}_{2}, 4 \mathrm{H}\right), 1.95\left(\mathrm{t}, J=0.8 \mathrm{~Hz}, \mathrm{COC}\left(\mathrm{C}_{3}\right)=\mathrm{CH}_{2}, 3 \mathrm{H}\right), 1.86(\mathrm{~d}, J$ $\left.=5.6 \mathrm{~Hz}, \underline{\mathrm{C}}_{3} \mathrm{CH}=\mathrm{CH}, 3 \mathrm{H}\right) ;{ }^{13} \mathrm{C} \mathrm{NMR}\left(100 \mathrm{MHz}, \mathrm{CDCl}_{3}\right) \delta 166.9$ and $166.7(\mathrm{C}=\mathrm{O}), 145.5$ ( $\left.\underline{\mathrm{CH}}=\mathrm{CHCO}\right)$, $139.6\left(\mathrm{CH}_{3} \mathrm{CH}=\underline{\mathrm{CH}}\right), 135.7\left(\mathrm{CO} \underline{\underline{C}}\left(\mathrm{CH}_{3}\right)=\mathrm{CH}_{2}\right), 129.5\left(\mathrm{CH}_{3} \underline{\mathrm{CH}}=\mathrm{CH}\right), 125.8\left(\mathrm{COC}\left(\mathrm{CH}_{3}\right)=\underline{\mathrm{CH}_{2}}\right), 118.0$ $(\mathrm{CH}=\underline{\mathrm{CHCO}}), 62.3\left(\mathrm{CH}_{2} \underline{\mathrm{C}}_{2}\right), 61.7\left(\mathrm{C}_{2} \mathrm{CH}_{2}\right), 18.4\left(\mathrm{COC}\left(\underline{\mathrm{CH}}_{3}\right)=\mathrm{CH}_{2}\right), 18.0\left(\underline{\mathrm{CH}}_{3} \mathrm{CH}=\mathrm{CH}\right)$.

(N-Hexa-2,4-dienoyloxy)-5-fluorourasyl (4e):

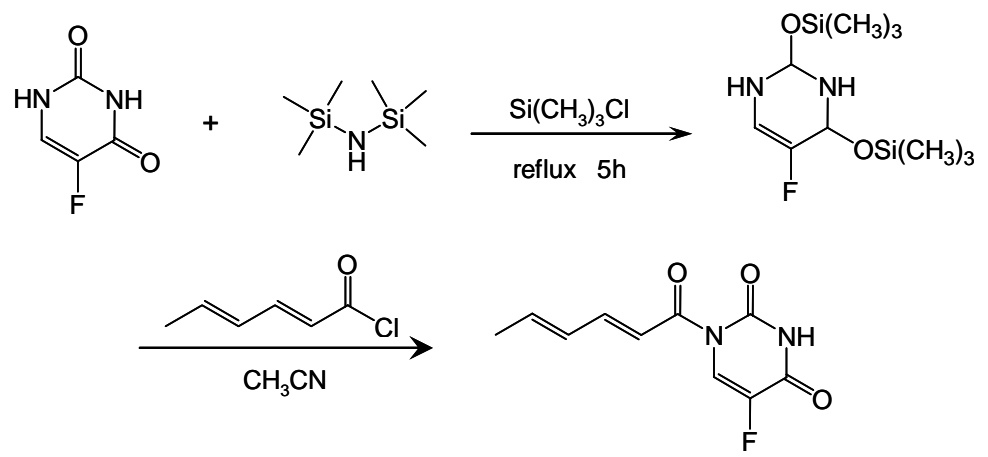

5-Fluorourasyl (4.0 g, $30 \mathrm{mmol}$ ) and catlytic amount of trimethylsilyl chloride were added to $15 \mathrm{~mL}$ of hexamethyldisilazane (HMDS), and the mixture was refluxed for $5 \mathrm{~h}$. After cooling to room temperature, any unreacted HMDS was removed under reduced pressure. The residue was dissolved in dry acetonitrile, and sorbic acid chloride (4.0 g) as an acetonitrile solution was dropwise added. After stirring overnight, a small amount of methanol was added. The solvent was evaporated and recryallization from acetone provided the product 4e. Yield 36.0\%.

4e: powder; mp 170-171 ${ }^{\circ} \mathrm{C}$; ${ }^{1} \mathrm{H}$ NMR $\left(400 \mathrm{MHz}, \mathrm{CD}_{3} \mathrm{OD}\right) \delta 8.29$ (d, $\left.J=7.2 \mathrm{~Hz}, \mathrm{C} \underline{\mathrm{H}}=\mathrm{CFCO}, 1 \mathrm{H}\right)$, 7.54 (m, $\underline{\mathrm{H}}=\mathrm{CHCO}, 1 \mathrm{H}), 7.07$ (d, $J=15.2 \mathrm{~Hz}, \mathrm{CH}=\mathrm{C} \underline{\mathrm{HCO}}, 1 \mathrm{H}), 6.43$ (m, $\left.\mathrm{CH}_{3} \mathrm{C} \underline{\mathrm{H}}=\mathrm{C} \underline{\mathrm{H}}, 2 \mathrm{H}\right), 1.91$ (d, $J=$ $\left.4.8 \mathrm{~Hz}, \mathrm{C}_{3} \mathrm{CH}=\mathrm{CH}, 3 \mathrm{H}\right) ;{ }^{13} \mathrm{C}$ NMR (100 MHz, $\left.\mathrm{CD}_{3} \mathrm{OD}\right) \delta 166.3(\mathrm{CH}=\mathrm{CHCO}), 159.4$ (d, $J=27.2 \mathrm{~Hz}$, $\mathrm{CH}=\mathrm{CF} \underline{C O}), 150.0(\mathrm{NCONH}), 149.6(\underline{\mathrm{CH}}=\mathrm{CHCO}), 144.1$ (d, $J=237 \mathrm{~Hz}, \mathrm{CH}=\underline{\mathrm{CFCO}}), 143.9$ $\left(\mathrm{CH}_{3} \mathrm{CH}=\underline{\mathrm{CH}}\right), 131.6\left(\mathrm{CH}_{3} \underline{\mathrm{CH}}=\mathrm{CH}\right), 124.0(\mathrm{~d}, J=37.1 \mathrm{~Hz}, \underline{\mathrm{CH}}=\mathrm{CFCO}), 121.4$ (CH=$\left.\underline{\mathrm{CHCO}}\right), 19.0$ $\left(\mathrm{CH}_{3} \mathrm{CH}=\mathrm{CH}\right)$. 
Sorbic acid azide (5a). Sorbic acid chloride $(50 \mathrm{mmol})$ was dropwise added while stirring to the suspension of sodium azide (3.6 g, $60 \mathrm{mmol}$ ) in 1,2-dichloroethane at $0^{\circ} \mathrm{C}$ over 30 min under nitrogen atmosphere. After stirring for 1 day, more sodium azide $(1.2 \mathrm{~g}, 20 \mathrm{mmol})$ was added to the reaction mixture, and then stirred for 1 day. The reaction mixture was filtered, and the filtrate was washed with $\mathrm{NaHCO}_{3}$, water, and brine. After drying, the solvent was evaporated under reduced pressure. Because the obtained $\mathbf{5 a}$ is an explosive compound, its purification was not carried out. The yield of the crude product 5 a was $63 \%$.

5a: ${ }^{1} \mathrm{H}$ NMR (400 MHz, $\left.\mathrm{CDCl}_{3}\right) \delta 7.35$ (dd, $J=9.6 \mathrm{~Hz}$, and $\left.15.2 \mathrm{~Hz}, \underline{\mathrm{C}}=\mathrm{CHCO}, 1 \mathrm{H}\right), 6.29$ (m, $\mathrm{CH}_{3} \mathrm{CH}=\mathrm{CH}$ and $\left.\mathrm{CH}_{3} \mathrm{CH}=\mathrm{C} \underline{H}, 2 \mathrm{H}\right), 5.79(\mathrm{~d}, J=15.2 \mathrm{~Hz}, \mathrm{CH}=\mathrm{CHCO}, 1 \mathrm{H}), 1.90(\mathrm{~d}, J=5.6 \mathrm{~Hz}$, $\left.\mathrm{C}_{3} \mathrm{CH}=\mathrm{CH}, 3 \mathrm{H}\right) ;{ }^{13} \mathrm{C}$ NMR $\left(100 \mathrm{MHz}, \mathrm{CDCl}_{3}\right) \delta 171.6(\mathrm{C}=\mathrm{O}), 146.7(\underline{\mathrm{CH}}=\mathrm{CHCO}), 141.9\left(\mathrm{CH}_{3} \mathrm{CH}=\underline{\mathrm{CH}}\right)$, $129.4\left(\mathrm{CH}_{3} \underline{\mathrm{CH}}=\mathrm{CH}\right), 119.7(\mathrm{CH}=\underline{\mathrm{CHCO}}), 18.5\left(\underline{\mathrm{C}} \mathrm{H}_{3} \mathrm{CH}=\mathrm{CH}\right)$.

$n$-Propyl $N$-(1,3-pentadienyl)carbamate (5c). 5a in 1,2-dichloroethane was refluxed for $5 \mathrm{~h}$. The obtained solution of sorbic acid isocyanate (1-isocyanato-1,3-pentadiene) $\mathbf{5 b}$ was cooled to room temperature, and triethylamine as the catalyst and excess 1-propanol were slowly added. After stirring for 1 day, the solution was evaporated and purified by recrystallization from a mixture of diethyl ether and $n$-hexane (1:1). The yield was $48.3 \%$.

5c: ${ }^{1} \mathrm{H}$ NMR (400 MHz, $\mathrm{CDCl}_{3}$ ) $\delta 6.65$ (dd, $J=11.6 \mathrm{~Hz}$ and $13.2 \mathrm{~Hz}, \mathrm{CH}=\mathrm{C} \underline{H N H}, 1 \mathrm{H}$ ), 6.35 (broad, $\mathrm{NH}, 1 \mathrm{H}$ ), 6.01 (dd, $J=11.6 \mathrm{~Hz}$ and $14 \mathrm{~Hz}, \mathrm{CH}_{3} \mathrm{CH}=\mathrm{C} \underline{\mathrm{H}}, 1 \mathrm{H}$ ), 5.67 (dd, $J=10.8 \mathrm{~Hz}$ and $13.6 \mathrm{~Hz}$, $\mathrm{CH}_{3} \mathrm{C} \underline{\mathrm{H}}=\mathrm{CH}, 1 \mathrm{H}$ ), 5.55 (m, $\left.\underline{\mathrm{H}}=\mathrm{CHNH}, 1 \mathrm{H}\right), 4.09$ (t, $\left.J=6.4 \mathrm{~Hz}, \underline{\mathrm{C}}_{2} \mathrm{CH}_{2} \mathrm{CH}_{3}, 2 \mathrm{H}\right), 1.74$ (d, $J=6.8 \mathrm{~Hz}$, $\left.\mathrm{C}_{3} \mathrm{CH}=\mathrm{CH}, 3 \mathrm{H}\right), 1.68$ (m, $\left.\mathrm{CH}_{2} \underline{\mathrm{C}}_{2} \mathrm{CH}_{3}, 2 \mathrm{H}\right), 0.96$ (t, $\left.J=7.6 \mathrm{~Hz}, \mathrm{CH}_{2} \mathrm{CH}_{2} \mathrm{C}_{3}, 3 \mathrm{H}\right) ;{ }^{13} \mathrm{C} \mathrm{NMR}(100 \mathrm{MHz}$, $\left.\mathrm{CDCl}_{3}\right) \delta 153.8(\mathrm{C}=\mathrm{O}), 128.6 \quad\left(\mathrm{CH}_{3} \mathrm{CH}=\underline{\mathrm{CH}}\right), 125.6 \quad\left(\mathrm{CH}_{3} \underline{\mathrm{CH}}=\mathrm{CH}\right), 124.5 \quad(\mathrm{CH}=\underline{\mathrm{CHNH}}), 111.4$

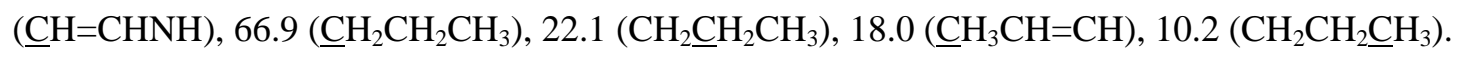

